\title{
High Prevalence Of Antibody Response Against Plasmodium Falciparum (Pf) Antigens In A Holoendemic Area Of Benin (1994-1995)
}

\section{S. Judith Gbenoudon Satoguina, (PhD)}

Laboratory of Immunology, Infectious and Allergic Diseases, ISBA and FAST, University of Abomey-Calavi, Cotonou, Benin

\section{Clemens H. Cocken, (PhD)}

Alan W. Thomas, (PhD)

Biomedical Primate Research Center, Delft, Holland

Jean Langhorne, (MD)

Imperial College of Science Technology and Medicine, London, England

Ambaliou Sanni, (PhD)

Laboratory of Biochemistry, Molecular Biology and Applications,

ISBA Cotonou University of Abomey-Calavi

\section{doi: 10.19044/esj.2016.v13n3p306 URL:http://dx.doi.org/10.19044/esj.2016.v13n3p306}

\begin{abstract}
The present study aimed at measuring the capacity of naturally occurring antibodies to bind Pf83/AMA-1 and MSP-1/19 antigens, two malaria vaccine candidates, in an immunoassay. According to the fact that antibody prevalence reflects endemicity of malaria, we further aimed at using the results obtained here as baseline data set to follow up and evaluate the expected decline in endemicity in 2016, 8 years after the change in drug policy in Benin. Therefore, individuals, 2 - 19 and above 20 years old, living in Awansori, a malaria holoendemic area in the suburb of Cotonou, Benin were bled during the dry and raining seasons of the years 1994/1995. Antibody responses were measured using direct, indirect and competition ELISA. We found a very high prevalence of antibody responses (89 to 96\%) in the studied population. The results indicate for Pf83/AMA-1, that naturally occurring antibodies bind to protective epitopes in a competition ELISA with a parasite inhibiting monoclonal antibody. The data and samples analysed here were collected during the rainy season 1994 and the following dry season 1994/1995.
\end{abstract}

Keywords: MSP-1/19 and Pf83/AMA-1 antigens, antibodies, ELISA, vaccine candidates 


\section{Introduction}

Malaria is a common infectious disease in tropical countries and particularly in Africa. More than half of the world population lives in the 103 countries where malaria is endemic (Alonso, 1994, Saklatvala 1993). The disease is induced by the Plasmodium genus, of which many species have been identified, in humans: $P$. falciparum, $P f, P$. vivax, $P$. ovale, $P$. malariae; with the mice: $P$. chabaudi $P$. yoeli; in rats, $P$. berghei, and in monkeys: $P$. Knowlesi. Among all, $P f$ is the most dangerous form of malaria in humans (Mendis, 1995). The disease control combines adequate treatment, vector control (Stephenson, 1991) and chemoprophylaxis in pregnant women. $P f$ has a complex cycle that brings into play two different hosts: man (intermediate host) and the female mosquito, anopheles (definitive host). It has a sexual lifecycle in the mosquito and an asexual one in humans (Deans, 1988). Throughout its lifecycle in humans, the parasite synthesizes different antigenic proteins, some of which are stage specific (Stephenson, 1991). AMA-1 and MSP-1 antigens are vaccine candidates from the Pf erythrocytic stage (Bouharoun- Tayoun, 1995) that are clearly immunogenic (GBENOUDON et al., 2016). Long-lasting antibodies against malaria in immigrants from several West African countries in Spain, were directed to AMA-1 and the $42 \mathrm{KD}$ piece of MSP-1 proteins (Moncunill G et al., 2013). Monoclonal antibodies directed against Pf83/AMA-1 inhibited the growth of Pf. Thus, anti Pf83/AMA-1 antibodies play an important role in the defense against Pf (Terrientes 1994).

The current study aimed at measuring naturally induced antibodies to the $83 \mathrm{KD}$ AMA-1 and $19 \mathrm{KD}$ MSP-1 antigens in individuals living in an endemic area, and at testing their ability to prevent the fixation of monoclonal antibodies directed against protective epitopes. Furthermore, we aimed at measuring the prevalence of antimalarial antibodies in the study population in order to document the decline of malaria in that same population, many years after the country treatment policy has changed for malaria.

Direct, indirect and competitive ELISA allowed measuring the recognition of Pf83/AMA-1 and MSP-1/19 antigens, alongside with the ability of naturally occurring antibodies to bind to protective epitopes. The results showed a high prevalence (89 to $96 \%$ ) of antibody response to the two antigens regardless of the seasons. It also indicated that anti-Pf83/AMA1 antibodies competed with the binding to protective Pf83/AMA-1 epitopes. These results show the holoendemic feature of the studied area at the time of sample collection. Most interestingly, the results are essential set of data for a comparative analysis of the data that are being collected in the same area throughout 2016. 
I.

\section{Material and methods}

We have collected blood samples in heparin tubes from 102 individuals (asymptomatic) living in Awansori in the outskirts of Cotonou, the economic capital of Benin, West Africa. The plasmas were conditioned with $1 \%$ azide and aliquots were stored at $-80^{\circ}$ Celsius until use. Parasitaemia at the time of sample collection, the spleen rate, age and sex, and malaria treatment were established during the samplings.

The antigens used came from 7G8 parasite strains, NF54 (CVD-1 clone), and the Welcome strain of Plasmodium falciparum (Pf) cloned and adapted for in vitro culture. The 7G8 strain was used for the preparation of Pf83/AMA-1 (83 KD fragment of the Pf apical membrane antigen), MSP-1/19 (19 KD C-terminal fragment of the Pf major merozoites protein) was obtained from the Welcome strain (Burghaus, 1994) and total parasite antigens (TPA) were obtained from the CVD-1 clone of the NF54 strain (Thomas, 1994).

Different ELISA methods were used for this study. Direct ELISA was used to measure out the isotypes of antibodies or immunoglobulins. For this purpose, the total antigens were bound on the ELISA plate for the night and the specific antibodies contained in plasmas detected according to the method described by Thomas et al. (Thomas, 1994).

For the determination of antibodies specific to Pf83/AMA-1 and MSP-1 antigens, a capture ELISA was used as previously described (Thomas, 1994). In short, monoclonal antibodies specific to these antigens were first bound on the ELISA plate, then the Pf83/AMA-1 and MSP-1/19 antigens were captured on these antibodies bound on the plate, before plasma addition.

For the competitive ELISA, it was intended to indirectly determine, if specific Pf83/AMA-1 antibodies contained in plasmas share the same epitopes as monoclonal antibodies directed against this same vaccine candidates, which inhibited the growth of Pf in vitro (Thomas, 1984). For this, the plates bound as previously described were filled either with single monoclonal antibodies or plasmas. Then, the plasmas-filled plates have been washed and filled again with monoclonal antibodies. Thus, only the Pf83/AMA-1 antigen sites that were not bound with the antibodies contained in the pre-incubated plasmas reacted with the monoclonal antibodies. The high-capacity binding 96-well plates (flat-bottomed microtiter ELISA plates, Greiner Labortechnik, Solingen, Germany) were used.

For the detection of specific antibodies contained in the tested plasma, the goat anti- human IgG antibody conjugated to alkaline phosphatase (Boehringer, Germany) was used, for the total IgG, and mouse anti human isotype antibodies, respectively, for the IgG $1,2,3,4$, and mouse 
anti-human IgM (Boehringer, Germany) for IgM detection, respectively. The purified human IgG, IgG1-4 antibody isotypes, and the purified IgM (all from Sigma, Germany) served as a positive standard for each antibody to be detected. We also used 28G2dcl and 4G2dc1 rat monoclonal antibodies that respectively recognize the Pf83/AMA-1 $\mathrm{C}$ and $\mathrm{N}$ terminus. The measurements have been carried out in the Immunology labs at BPRC, Delft, Netherlands and at the Imperial College, London, England.

\section{Data analysis}

The average value of the OD measured was obtained through the arithmetic mean of duplicate wells. We calculated a geometric mean, a standard deviation from the negative plasmas, then the ELISA Index (EI) using the formula as follows:

$E I=$ arithmetic mean (duplicate wells of tested plasma) / [(geometric mean (negative controls) + 3 (Standard Deviation)]

The coefficient of variation (CV) is calculated for the whole test and as follows: $C V=$ [standard deviation (OD negative controls) / geometric mean (OD negative control)] $\times 100$

For the competitive inhibition ELISA, the remaining binding activity of the monoclonal 4G2-AP was obtained by dividing the specific OD (arithmetic mean) by that of the positive control (4G2-AP only). The inhibition percentage (\% Inhibit) could then be calculated as follows:

$\%$ Inhibit. $=100 \times(1-[($ arithmetic mean OD plasma test $) /$ (arithmetic mean OD positive control)]

\section{Results}

\section{Parasitaemia in the study population}

Individuals $(n=100)$, divided into 5 age groups (Diagram 1 ) were taken into account in this study. The parasite rate at the time of sample collection was $30 \%$ in the dry season (12 830 parasites/ $\square$ l of blood on average) and 35\% in the humid season (an average of 1750 parasites/ $\square$ l of blood). The parasitaemia, expressed as the number of parasites / $\square$ l of blood, was decreasing with age. Minimum/maximum parasitaemia values were 100/54000 and 100/14000 respectively in dry and humid seasons.

\section{Prevalence of IgG antibodies specific to Pf83/AMA-1 and MSP-1/19 antigens}

Both antigens gave high prevalence on average, 96\% for IgG antibodies during the dry season. This prevalence remained during the humid season for Pf83/AMA-1 and with slight decrease for MSP-1/19 (89\%). Specific antibodies to total parasite antigens (TPA) were rather lower and prevalence rates here were constant in both seasons across the years. The 
prevalence rate for the AMA-1 was increasing with age up to 10 years old. For the other age groups, the rate increased up to $100 \%$ and remains the same during the seasons. Such distribution was not seen for MSP1/19. Regarding Pf83/AMA-1 and MSP-1/19, there is a clear difference between the prevalence rate of the 2-4 years age group and other age groups (Figure 1). The lowest IgG rates were observed within the 2-4 years age group. These results proved that there is a good correlation between age and anti-AMA1 and MSP-1/19 antibody response. For MSP-1/19 however, the high prevalence was already observed in 2-4 years (dry season, figure 1). Prevalence generally increased in the subsequent age groups.

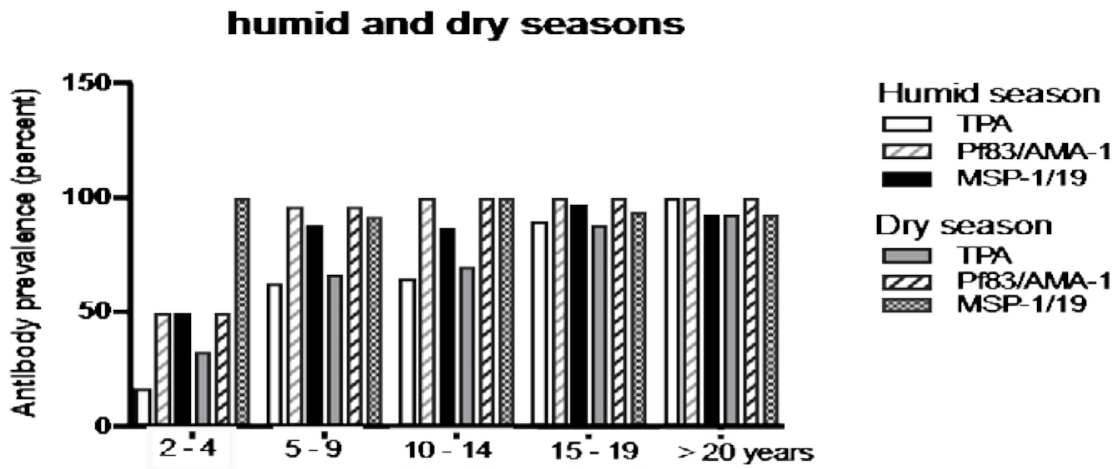

Figure 1. Age group dependant prevalence of antibodies to Pf83/AMA-1 and MSP-1/19 and to total Pf antigens

The total IgG specific to TPA, Pf83/AMA-l, and MSP-1/19 antigens respectively, were determined by ELISA in the indicated age groups. Plasma were tested at a dilution of $1 / 200$. The total IgG bound to antigens were detected by the goat anti-human IgG antibody conjugated to alkaline phosphatase (IgG-AP) and PNPP substrate. Optical densities (OD) read at $405 \mathrm{~nm}$ were then converted to ELISA index (EI) using the negative controls on each plate (see methods). The number of antibody-positives i.e. El $\geq 1$ was counted for each season in each age group and for the total of 100 samples and expressed as a percentage (\%).

\section{Isotypes Antibody Response}

Of the IgG isotypes tested, IgG1 antibody against Pf83/AMA-1 antigen was predominant in all age groups, the rate of IgG1 increased from 2-4 years with a peak at the 15-19 years age group (Figure 2.A). For MSP1/19 (Figure 2.B), there was an IgM predominance in children of 2-4 years. For the remaining groups, IgG1 was predominant. IgM response was also prevalent against total parasite antigens (TPA), Diagram 2.C 

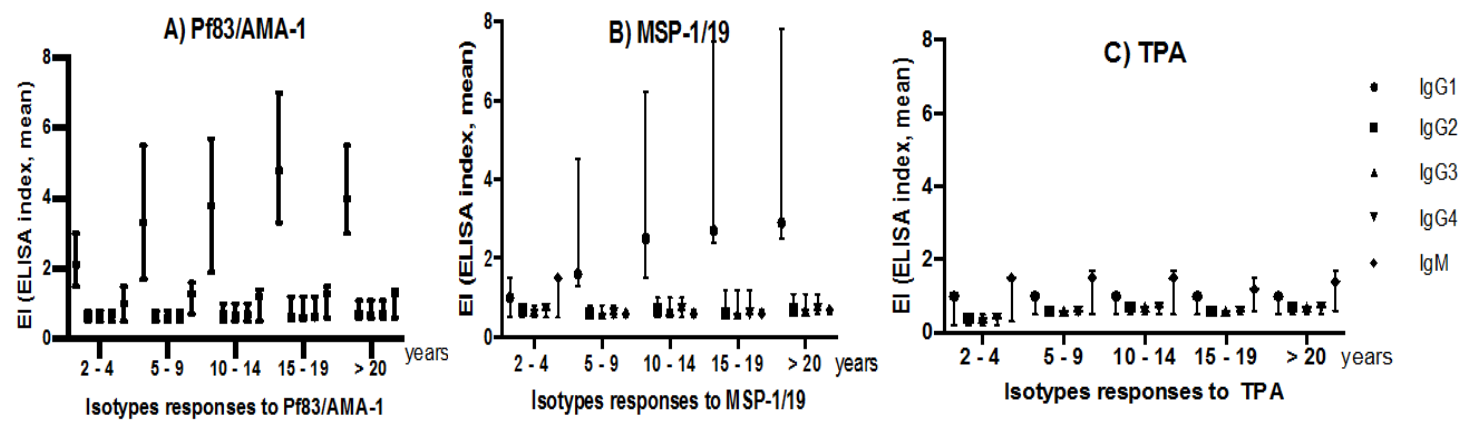

Figure 2. Isotypes antibodies response to the antigens

IgGl, IgG2, IgG3, IgG1 to 4, and IgM Isotypes specific to A) Pf83/AMA-l, B) MSP-1/19, and C) TPA antigens respectively, were determined by ELISA in 100 (one hundred) individuals belonging to specific age groups as indicated. Plasma were tested at $1 / 200$ dilution. The isotypes were detected by a mouse anti-human antibody, conjugated with alkaline phosphatase (IgG-AP), and PNPP substrate. Optical densities (OD) read at $405 \mathrm{~nm}$ were then converted to ELISA index (EI). The geometric mean of El was calculated for each age group as well as the corresponding standard deviation. Each curve represents a particular isotype. Vertical bars represent the standard deviation values of each group.

Plasma actively competed with the monoclonal antibody (that inhibits the Pf growth) for binding on the antigen

Pf83/AMA-1 inhibition test was conducted and inhibition rates of at least $10 \%$ was recorded in $97 \%$ of individuals; inhibition rates of $\geq 50 \%$ were obtained in 82 and $79 \%$ of individuals, respectively for the dry and humid seasons. The distribution of inhibitions within age groups was the same across seasons.

Table 1. Inhibition of the fixation of parasite growth inhibiting monoclonal antibody (\%) per age group

\begin{tabular}{ccc}
\hline Age group (year) & Dry season & Humid season \\
\hline $2-4(\mathrm{n}=6)$ & 50 & 50 \\
$5-9(\mathrm{n}=24)$ & 83 & 83 \\
$10-14(\mathrm{n}=23)$ & 79 & 79 \\
$15-19(\mathrm{n}=33)$ & 94 & 88 \\
$\geq 20(\mathrm{n}=14)$ & 71 & 64 \\
Total $(\mathrm{n}=100)$ & 82 & 79 \\
\hline
\end{tabular}




\section{Discussion}

\section{IgG antibody responses directed against Pf83/AMA-1 and MSP-119 antigens}

We measured the specific antibodies naturally directed against Pf83/AMA-l and MSP-1/19 and found that 96\% of surveyed individuals had antibodies against both antigens. Pf83/AMA-1 and $M S P-1 / 19$ antigens were recognized by the plasmas of naturally exposed individuals, some of which were actively infected although asymptomatic (30-35\% depending on the season). These results are consistent with those obtained in Guinea-Bissau and in a highly endemic region of Senegal with respect to Pf83/AMA-1 (Peterson, 1989). IgG predominance in these regions ranged from 94 to $100 \%$ of surveyed individuals (Riley, 1992). In two different regions of Gambia (West Africa), where malaria is seasonal, antibodies directed against MSP-1 were also found (Riley, 1992). Even nowadays antibodies against AMA-1 are still prevalent in endemic area, expectedly however, in much lower extend (Gbenoudon Satoguina et al., 2016, Moncunill et al., 2013, Satoguina et al., 2009; Aucan et al., 2001). In The Gambia, the prevalence was sometimes much lower (40\%) than the one obtained in the current study at similar periods (Riley et al., 1992). This may be linked to the specificity of the area hereby surveyed where malaria transmission is perennial.

The prevalence of antibodies against Pf83/AMA-1 in individuals living in two endemic areas has also been already reported (Peterson, 1989). Several studies also reported the antibody response directed against different regions of the MSP-1 protein (Egan, 1995 Riley, 1992; Terrientes 1994, Akpogheneta OJ et al., 2007; Manz RA et al., 2005). In the present study we used two clearly defined purified recombinant proteins. The outcomes confirm that the proteins studied were immunogenic within the surveyed population in Benin, and help to document a period of high exposure to malaria at least in that area. We also had a lower prevalence rate regarding total parasite antigens (76\%) within the surveyed population. The simultaneous exposure of several epitopes may have hidden from an optimal binding.

There was an age-correlation for the responses to Pf83/AMA-l and MSP-1/19 proteins. Regarding MSP-1/19, a threshold is not reached in the surveyed age groups, however concentration of antibodies directed against Pf83/AMA-l increased with age up to a peak between 15 and 19 years. Age distribution observed with MSP-1/19 is very similar to the one observed with the total parasite antigens. These outcomes are consistent with those obtained in Colombia and in The Gambia (Terrientes 1994 Riley, 1992) where the anti-MSP 1/19 prevalence depended on age. In Guinea, a similar result was obtained for distribution according to age in response to Pf83/AMA-l. In Senegal, Pf83/AMA-l response was not age-dependent (Peterson, 1989). In 
endemic areas, protection against malaria is as age-dependent as are antibody responses so that mortality is restricted to non-immune individuals (Bouharoun- Tayoun, 1990, Früh 1991, Langhorne J et al., 2008).

Regarding the nature of the antibody isotype responses to MSP-1/19 and Pf83/AMA-l antigens, we found that IgG1 was predominant, followed by IgM. However the studied plasmas were generally negative for IgG2, IgG3 and IgG4. The antibody isotypes involved in the humoral response to $P f$ were evidenced by other authors who demonstrated the importance of the IgG1 and IgG3 isotypes in the development of protective immunity against malaria (Kinyanjui SM et al., 2007; Aubouy A et al., 2007). The study of isotypes produced in response to MSP-l /19 has been compared with results similar to those obtained. IgG1 was found predominant (Egan, 1995, Gbenoudon et al., 2016). Other studies using total parasite antigens also reported the predominance of IgG1 and IgG3 even in individuals subjected to repeated infections (Wahlgren, 1983 and 1986). Could the poor rate of IgG3 antibodies in our study be a particular feature of the study area? It was suggested that the IgG1 and IgG3 isotypes (cytophilic antibodies) would be predominant among pre-immune individuals while IgG2 and IgG4 (less cytophilic) and IgM could be present in non-immune individuals (Bouharoun-Tayoun, 1992). Our outcomes, with regard to IgG3, were not consistent with those obtained elsewhere.

\section{Inhibition of the binding of Pf83/AMA-1 specific monoclonal antibodies}

To study the fine feature of naturally acquired antibodies directed against Pf83/AMA-l, the plasma of the individuals of Awansori (the study area), a malaria holoendemic area in Benin, were tested against a negative plasma for the competitive inhibition of the binding by Pf83/AMA-1 specific monoclonal antibody (4G2dc1) to its epitope. The 4G2dc1 monoclonal antibody inhibits the parasite growth in vitro. Plasma inhibition of 4G2dc1 increased with plasma concentration thus was dose-dependent unlike negative plasma, which showed no inhibition. This means that there is competition on the monoclonal antibody binding site on the Pf83/AMA-1 with antibodies from the plasma of individuals exposed and/or infected. We found that $82 \%$ of the individuals surveyed inhibited at $\geq 50 \%$, the binding of monoclonal antibodies to Pf83/AMA-1. This indicates a certain prevalence of antibodies directed against similar epitopes in the studied plasma. This prevalence of inhibiting antibodies in the plasma, suggested that the epitope recognized by the monoclonal antibody and the antibodies contained in the plasma were very close to each other or alike. Inhibition also happened according to age. Similar inhibition was also reported with regard to the MSP-1/19 antigen (Egan, 1995). However, it should not be ruled out that the 
presence of plasma antibodies on Pf83/AMA-l antigenic molecule might also have prevented the binding of the monoclonal and lead to inhibition.

\section{Conclusion}

In conclusion, we studied the specific humoral immune response of MSP-1/19 and Pf83/AMA-l vaccine candidates in a group of 100 individuals living at Awansori, a holoendemic area in the outskirts of Cotonou in Benin. People were sampled during the humid and the dry seasons of 1994 and 1995, respectively. The results showed that:

- there was a predominance of IgG antibody response with IgG1 being the predominant isotype

- IgG and IgG1 were age-dependent

- The antibodies of studied plasma competitively inhibit the binding of the Pf83/AMA-l specific monoclonal 4G2dc1 antibody that inhibits the parasite growth in vitro. This suggests that the plasma recognized similar epitopes.

The present study will serve documenting the immuno-endemic parameters of an holoendemic area, at a time, where the malaria control programs were of a smaller scale, particularly regarding the use of efficient drugs and the nowadays widespread use of mosquito nets. On going studies, in 2016, in the same area will directly measure the impact of new antimalarial strategies by using the present study data as a baseline data set.

\section{Acknowledgement}

We thank Marius ADJAGBA, Magloire GBAGUIDI for samples collection and Anne Marie Voorberg Van der Wel for special laboratory assistance.

\section{References:}

1. Akpogheneta OJ, Tetteh KK, Dunyo S, Lanar DE, and Conway DJ. (2007) Duration of Naturally Acquired Antibody Responses to Blood-Stage Plasmodium falciparum Is Age Dependent and Antigen Specific. Infection and Immunity 76(4):1748-1755.

2. Alonso, P. L., Smith, T., Armstrong Schellenberg, J. R. M., Masanja, H., Mwankusye, S., Urassa, H., Bastos de Azevedo, 1., Chongela, J., Kobero, S., Menendez, C., Hurt, N., Thomas, M. C., Lyimo, E., Weiss, N. A., Hayes, R., Kitua, A. Y., Lopez, M. C., Kilama, W. L. and Teuscher, T. (1994) Randomised trial of SPf66 vaccine against Plasmodium falciparum malaria in children in southern Tanzania. Lancet 344: 11751181

3. Aubouy A, Migot-Nabas F, Deloron P.: Correlations between treatment outcome and both anti-MSP119 antibody response and 
erythrocyte-related genetic factors in Plasmodium falciparum malaria. Infect Genet Evol 2007, 7:147-154.

4. Aucan C, Y. Traore, F. Fumoux, and P. Rihet: Familial correlation of immunoglobulin $G$ subclass responses to Plasmodium falciparum antigens in Burkina Faso. Infect Immun 2001, 69:996-1001.

5. Bouharoun- Tayoun, H., Oeuvray,c., Lunel, F. and Druilhe, P. (1995) Mechanisms underlying the monocyte-mediated antibody-dependent killing of Plasrrwdium falciparum asexual blood stages. J. Exp. Med. 182: 409-418.

6. Bouharoun-Tayoun, H. and Druilhe, P. (1992) Plasmodium falciparum malaria: evidence for an isotype imbalance which may be responsible for delayed acquisition of protective immunity. Inf. and Immun. 60 (4): 1473-1481

7. Bouharoun- Tayoun, H., Attanath, P., Sabchareon, A., Chongsuphajaisiddhi, T., and Druilhe, P. (1990) Antibodies that protect humans against Plasmodium falciparum blood stages do not on their own inhibit parasite growth and invasion in vitro, but act in cooperation with monocytes. J. Exp. Med. 172: 1633-1641.

8. Burghaus, P. A. and Holder, A. A. (1994) Expression of the $19-\mathrm{kD}$ C-terminal fragment of the Plasmodium falciparum merozoite surface protein-l in E. coli as a correctly folded protein. Mol. and Biochem Parasitol. 64: 165-169.

9. Deans, J. A., Knight, A. M., Jean, W. C., Waters, A. P., Cohen, S. and Mitchell, G. H. (1988) Vaccination trials in rhesus monkeys with a minor, invariant, Plasmodium knowlesi 66kD merozoite antigen. Parasite Immunol. 10: 535-552.

10. Egan, A. F., Chappel, J. A., Burghaus, P. A., Morris, J. S., McBride, J. S., Holder, A. A., Kaslow, D. C. and Riley, E. M. (1995) Serum antibodies from malaria-exposed people recognize conserved epitopes formed by the two epidermal growth factor motifs of MSP$1 / 19$, the C-terminal fragment of the major merozoite surface protein of Plasmodium falciparum. Infect Immun. 63(2):456-66.

11. Früh, K., Doumbo, O., Müller, H.-M., Koita, O., McBride, J., Crisanti, A., Touré, Y. and Bujard, H. (1991) Human antibody response to the major merozoite surface antigen of Plasmodium falciparum is strain specific and short-lived. Infect Immun. 59 (4): 1319-1324.

12. Gbenoudon SJ, Kouakanou L, Degbelo JE, Deh-Tchokpon J, Conway D and Walther M. (2016) Induction d'immunoglobulines anti P. falciparum pendant le paludisme et après rémission chez les enfants au Benin. Le Journal de la Société de Biologie Clinique du Bénin. 
13. Howard, R. F. and Reese, R. T. (1984) Synthesis of merozoite proteins and glycoproteins during the schizogony of Plasmodium falciparum. Mol. and Biochem. Parasitoi. 10: 319-334.

14. Kinyanjui SM, Conway DJ, Lanar DE, and Marsh K. (2007) IgG antibody responses to Plasmodium falciparum merozoite antigens in Kenyan children have a short half-life. Malar J. 6:82.

15. Langhorne J, Ndungu FM, Sponaas AM, Marsh K. (2008) Immunity to malaria: more questions than answers. Nat Immunol. 9(7):725-732.

16. Manz RA, Hauser AE, Hiepe F, and Radbruch A. (2005) Maintenance of serum antibody levels. Annu Rev Immunol. 23:367386.

17. Mendis, K. N. and Carter, R. (1995) Clinical disease and pathogenesis in malaria. Parasitol. Today 11 (5): 1-16.

18. Müller, B.-M., Früh, K., Brunn, A., Esposito, F., Lombardi, S., Crisanti, A. and Bujard, H. (1989) Development of the human immune response against the major surface protein MSP-1I30(gpI90) of Plasmodium falciparum. Inf. and Immun. 57 (12): 37653769

19. Moncunill G, Mayor A, Jiménez A, Nhabomba A, Casas-Vila N, Puyol L, et al. (2013) High Antibody Responses against Plasmodium falciparum in Immigrants after Extended Periods of Interrupted Exposure to Malaria. PLoS ONE 8(8): e73624. doi:10.1371/journal.pone.0073624

20. Peterson, M. G., Marshall, V. M., Smythe, J. A, Crewther, P. E., Lew, A, Silva, A., Anders, R F. and Kemp, D. J. (1989) Integral membrane protein located in the apical complex of Plasmodiumfalciparum. Mol. and Celle Biol. 9 (7): 3151-3154.

21. Riley, E. M., Allen, S. J., Wheeler, 1. G., B1ackman, M. J., Bennett, S., Takacs, B., Schonfe1d, H.-J., Ho1der, A. A. and Greenwood, B. M. (1992) Naturally acquired cellular and humoral immune responses to the major merozoite surface antigen (Pf MSP1) of Plasmodium falciparum are associated with reduced malaria morbidity. Parasite Immunol. 14: 321-337.

22. Saklatvala, T. (1993) The management of drug resistance. Parasitol.Today. 9 (5): 149.

23. Satoguina J, Walther B, Drakeley C, Nwakanma D, Oriero EC, Correa S, Corran P, Conway DJ, Walther M. (2009) Comparison of surveillance methods applied to a situation of low malaria prevalence at rural sites in The Gambia and Guinea Bissau. Malar J. 8:274.

24. Siddiqui, W. A., Tarn, L. Q., Kramer, K., J., Hui, G. S. N., Case, S. E., Yamaga, K. M., Chang, S. P., Chan, E. B. T. and Kan, S.-C. (1987) Merozoite surface coat precursor protein comp1etely protects 
Aotus monkeys against Plasmodium falciparum malaria. Proc. Natl. Acad. Sci. USA. 84: 3014-3018.

25. Stephenson, M. M. (1991) In "Malaria: host responses to infection". M.M. Stephenson ed. CRC Pres, Inc. Boca Raton, Florida

26. Terrientes, Z., Kramer, K., Herrera, M., A, Chang, S., P. (1994) Naturally acquired antibodies against the major merozoite surface coat protein (MSP-1) of Plasmodium falciparum acquired by residents in an endemic area of Colambia. Men Inst Oswaldo Cruz, Rio de janeiro 89 (suppL Il): 55-61

27. Thomas, A W., Trape, J. E, Rogier, C., Goncalves, A, Rosario, V. E. and Narum, D. L. (1994) High prevalence of natural antibodies against Plasmodiumfalciparum $83-\mathrm{kD}$ apical membrane antigen (Pf 83/AMA-1) as detected by capture-enzyme -linked immunosorbent assay using full-Iength baculovirus recombinant Pf 83/ AMA-1. Am. J. Trop. Med. Hyg. 51 (6): 730-740.

28. Thomas, A. W., Deans, J. A., Mitchell, G. H., Alderson, T. and Cohen, S. (1984) The Fab fragments of monoclonal IgG to a merozoite surface antigen inhibit Plasmodium knowlesi invasion of erythrocytes. Mol. and Biochem. Parasitoi. 13: 187-199

29. Wahlgren, M., Berzins, K., Perlmann, P. and Persson, M. (1983) Characterization of the humoral immune response in Plasmodium falciparum malaria. IL IgG subclass 1eve1s of anti-P.falciparum antibodies in a different sera. Clin. Exp. Immunol. 34: 135-142.

30. Wahlgren, M., Perlmann, H., Berzins, K., Bjorkman, A., Larsson, Â., Ljungstrom, 1., Patarroy, M. E. and Perlmann, P. (1986) Characterization of the humoral immuneresponse in Plasmodium falciparum malaria. ill. Factors influencing the coexpression of antibody isotypes (IgM and IgG-1 to 4). Clin. Exp. Immunol. 63: 343353 